\title{
Synthesis of the 2'-Azidoethyl Trisaccharide, 6d-altroHepp-GlcNAc-Gal Hapten, an $O$-Antigenic Repeating Unit of Campylobacter jejuni Serotypes O:23 and O:36
}

\author{
Mikyung Yun ${ }^{+* a}$ and Jeong E. Nam Shin \\ ${ }^{\dagger}$ Deparment of Applied Chemistry Donguang Technical College, Seoul 152-714, Korea \\ Department of Chemistry, Soongsil Lniversity, Seoul 156-7+3, Korea. E-mail: namiamail.ssu.ac.kr \\ Received Jahuarv 30, 2008
}

\begin{abstract}
A trisaccharide. $6 \mathrm{~d}-1 /$ tro-Hepp $\alpha(1 \rightarrow 3) \mathrm{GlcNAc} \beta(1 \rightarrow 3) \mathrm{Gal} \alpha\left(1 \rightarrow O \mathrm{CH}_{2} \mathrm{CH}_{2} \mathrm{~N}_{2}\right.$. as an $O$-antigenic repeating unit of Campulobacter jejtmi serotypes $\mathrm{O}: 23$ and $\mathrm{O}: 36$. was synthesized. Coupling of the 6d-altro-Heppa $(1 \rightarrow 3) \mathrm{GlcNAc} \beta\left(1 \rightarrow\right.$ SEt donor with $\mathrm{Gal} \alpha\left(1 \rightarrow O \mathrm{CH}_{2} \mathrm{CH}_{2} \mathrm{Cl}\right.$ acceptor in the presence of NIS-TfOH promoter afforded the trisaccharide having the $\beta(\mathrm{l} \rightarrow 3) \mathrm{Gal}$ linkage. $\beta$-Stereospecificity and the desired regioselectivity for the $3-\mathrm{OH}$ Gal are obtained. Subsequent hydrogenation. acetylation. azide displacement. hydrazinolysis. $\bar{X}-$ acetỵlation and finally deacetylation fumished the title trisaccharide hapten for further glycoconjugation.
\end{abstract}

Key Words : Compwobacter jejumi. Trisaccharide. 6d-altroHepp-GlcNAc-Gal hapten. O-Antigenic repeating unit

\section{Introduction}

Campylobacter jejuni a species of curved. motile and Gram-negative bacilli. is recognized as one of the conmonest causes of infective diarrhea and acute bacterial enteritis. Campy lobacter infection has been reported to be associated with neuropathy' known as Guillain-Berre syndrome (GBS) and Miller-Fisher sy'ndrome (MFS) ${ }^{1.2}$

lnvestigations on their chemical structures by Aspinall et $a .^{3-6}$ showed that $C$. jeituni serotypes $0: 23$ and $0: 36$ possess high-molecular weight LPS $O$-glycans which are crossreacting. The $O$-glycans. a potential basis of the serological classification of $C$. jejumi are consisted of trisaccharide repeating units having unusual altroheptose residues which vary in the presence or absence of oxygenation at C-6 and methylation at $O-3$ from batch to batch. The structural difference in the heptose components may constitute a basis for serotypic discrimination. or evading the immune response of the host. In order to elucidate the role of altroheptose residues and evaluate the different reading frames in the immunological specificity: it was necessary to synthesize various oligosaccharides containing the repeating unit of the C. jejuni serotypes $\mathrm{O}: 23$ and $0: 36^{6-9}$ Three trisaccharides of different reading frames with the repeating sequence have been synthesized. ${ }^{1611}$ Herein. we wish to describe a synthesis of the trisaccharide. 6d-Altro-Heppo $(1 \rightarrow 3)$ GlcNAc $\beta$

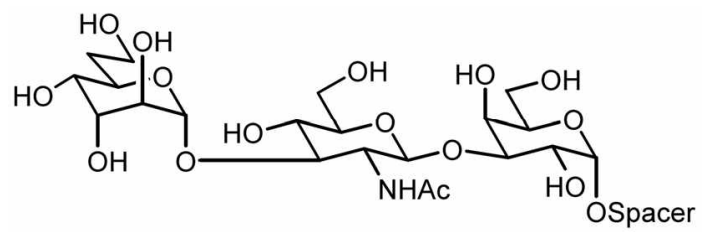

Figure 1. O-Antigenic Polvsaccharide of Camprlobacter jejuni $\mathrm{O}$ : 23 and $0: 36$

"Present address: Division of Liberal Arts and Teacher's Education. University of Seoul. Seoul 130-743. Korea
$(1 \rightarrow 3)$ Gal $\alpha$ in the fonn of its 2'-azidoethyl glycosides (Figure 1). The azide group was introduced to the trisaccharide as a linker to a peptide. which would enable to evaluate the trisaccharide as an immunogen

\section{Results and Discussion}

For the synthesis of the desired trisaccharide 1, the two building blocks. 6d-altro-Hepp $\alpha(1 \rightarrow 3) \mathrm{GlcNPhth} \beta(1 \rightarrow \mathrm{SEt}$ donor 7 and $\mathrm{Gal} \alpha\left(1 \rightarrow \mathrm{OCH}_{2} \mathrm{CH}_{2} \mathrm{Cl}\right.$ acceptor 6. were first synthesized. as shown in Schemes 1 and 2.

The $6 \mathrm{~d}$-altro-Hepp $\alpha(1 \rightarrow 3)$ GleNPhth 7 has been prepared from 6d-manno-Hepp $\alpha(1 \rightarrow 3)$ GlcNPhth by Sitern oxidation $^{12}$ and subsequent reduction as described previously. ${ }^{1 !}$ For the synthesis of the Gal acceptor 6. ethylthio 2.6-di- $O$ benzyl-3.4- $O$-isopropylidene- $\beta$-D-galactopyranoside 3 was prepared by acetonation of ethylthio $\beta$-D-galactopyranoside with dimethoxypropane and 4-toluenesulfonic acid in THF ( $73 \%$ yield). and the subsequent benzylation with benzyl bromide in DMF (a quantitative yield). The fully protected ethylthio $\beta$ D-galactopyranoside 3 was coupled to $2-$ chloroethanol in the presence of IDC $\mathrm{P}^{1(t) 3}$ giving the $\alpha$ - and $\beta-2^{\prime}$-chloroethyl galactoside 4 and 5 in ratio of $5.1 / 1$ in $65 \%$ vield. The use of NIS-TfOH ${ }^{1+15}$ promoter decreased the stereoselectivity of $\alpha / \beta$ to $1.7 / 1$, while increasing the coupling yield to $94 \%$. The $3.4-O$-isopropylidene group in $\mathbf{4}$ was deacetonated with a catalytic amount of $p$-toluenesulfonic acid and gave the 2'-chloroethyl galactoside donor 6 which has free 3- and 4-OH groups (Scheme 1).

The coupling of the altroHep-GlcNPhth disaccharide donor 7 and Gal acceptor 6 in the presence of NIS-TfOH ${ }^{14.15}$ affored the trisaccharide. 2'-chloroethyl $O$-(3-O-acetyl-7-Obenzoyl-2.4-di- $O$-benzyl-6-deosy- $\alpha$-D-altroheptopyranosyll)$(1 \rightarrow 3)$-(4.6-di-O-acetyl-2-deoxy-2-phthalimido- $\alpha$-D-glucopyranosyl)-(l $\rightarrow 3$ )-2.6-di- $O$-benzyl- $\beta$-D-galactopyranoside $\mathbf{8}$ in $64 \%$ yield. The trisaccharide 8 showed the desired $\beta$ stereospecificity and the regioselectivity for the $3-\mathrm{OH}$ group of the Gal acceptor. After coupling, the downfield shift of 

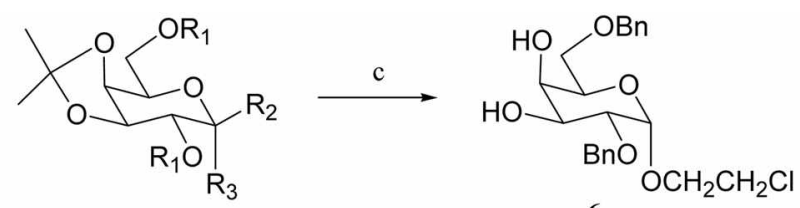

$$
\begin{aligned}
\text { 2. } \mathrm{R}_{1}=\mathrm{H}, \mathrm{R}_{2}=\mathrm{SEt}, \mathrm{R}_{3}=\mathrm{H} \\
\text { b } \longrightarrow \text { 3. } \mathrm{R}_{1}=\mathrm{Bn}, \mathrm{R}_{2}=\mathrm{SEt}, \mathrm{R}_{3}=\mathrm{H} \\
\text { 4. } \mathrm{R}_{1}=\mathrm{Bn}, \mathrm{R}_{2}=\mathrm{H}, \mathrm{R}_{3}=\mathrm{OCH}_{2} \mathrm{CH}_{2} \mathrm{Cl} \\
\text { 5. } \mathrm{R}_{1}=\mathrm{Bn}, \mathrm{R}_{2}=\mathrm{OCH}_{2} \mathrm{CH}_{2} \mathrm{Cl}_{2} \mathrm{R}_{3}=\mathrm{H}
\end{aligned}
$$

Scheme 1. Synthesis of the acceptor 6: (a) 1) NaH, DMF, $0^{\circ} \mathrm{C}, 40$ min, 2) $\mathrm{BnBr}$, 1t, lh, quntitative: (b) 2-chloroethanol, $\mathrm{DCP}, \mathrm{CH}_{2} \mathrm{Cl}_{2-}$ $\mathrm{Et}_{2} \mathrm{O}(2 / 5, v / \mathrm{v}), \mathrm{MS} 5 \AA$, rt, $30 \mathrm{~min}, 54 \%$; (c) $p$-TsOH, MeOH, 65 ${ }^{\circ} \mathrm{C}, 40 \mathrm{~min}, 91 \%$.

the C. -3 value of the galactosyl residue from 69.1 to 79.8 ppm has been observed, while the $\mathrm{C}-4$ value remained at $68.9 \mathrm{ppm}$ in the ${ }^{13} \mathrm{C}-\mathrm{NMR}$ spectrum. This confirms the formation of the $1 \rightarrow 3$ linked $\beta$-galactosyl trisaccharide (Scheme 2). The formation of the $\mathrm{I} \rightarrow+$ linked $\beta$-galactosyl trisaccharide was also observed in its ${ }^{13} \mathrm{C}$-NMR spectrum. The $1 \rightarrow+$ linked trisaccharide has not been identified further. Such a regioselectivity was not achieved in the previous synthesis. i.e., in the coupling of an $\alpha$-Gal acceptor having free $2-\mathrm{OH}$ and $3-\mathrm{OH}$ groups. where $1 \rightarrow 2$ and $1 \rightarrow 3$ linked trisaccharides were obtained in a $1: 1$ ratio. ${ }^{15.17}$

The trisaccharide 6d-altr $/ \mathrm{Hep}-\mathrm{GlcNPhth}-\mathrm{Gal}-\mathrm{OCH}_{2} \mathrm{CH}_{2} \mathrm{Cl}$ 8 was hydrogenated with $\mathrm{H}_{2}, \mathrm{Pd} / \mathrm{C}$ and then acetylated to give 11. During the hydrogenation dechlorination accompanied and gave ethyl glycoside $\mathbf{1 2}$ as a minor product. The tri. saccharide 11 and its side product 12 were not separated, but

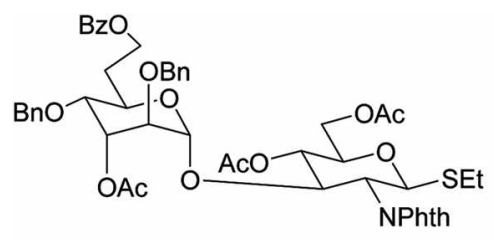

7

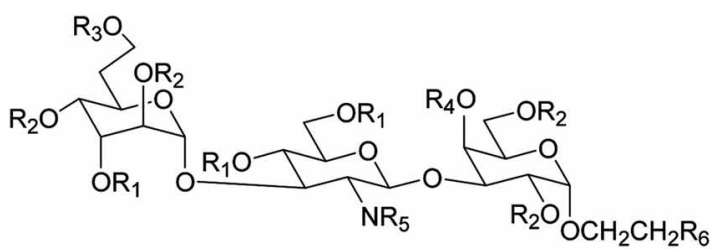

b - 8. $R_{1}=A c, R_{2}=B n, R_{3}=B z, R_{4}=H, R_{5}=P h t h, R_{6}=C l$

9. $\mathrm{R}_{1}=\mathrm{Ac}, \mathrm{R}_{2}, \mathrm{R}_{4}=\mathrm{H}, \mathrm{R}_{3}=\mathrm{Bz}, \mathrm{R}_{5}=\mathrm{Phth}, \mathrm{R}_{6}=\mathrm{Cl}\left(10 . \mathrm{R}_{6}=\mathrm{H}\right)$

11. $R_{1}, R_{2}, R_{4}=A c, R_{3}=B z, R_{5}=P h t h, R_{6}=C l\left(12 . R_{6}=H\right)$

P 13, $\mathrm{R}_{1}, \mathrm{R}_{2}, \mathrm{R}_{4}=\mathrm{Ac}, \mathrm{R}_{3}=\mathrm{Bz}, \mathrm{R}_{5}=$ Phth, $\mathrm{R}_{6}=\mathrm{N}_{3}\left(14, \mathrm{R}_{6}=\mathrm{H}\right)$

$f=15 \cdot R_{1}, R_{2}, R_{3}, R_{4}=A c, R_{5}=H A c, R_{6}=N_{3}\left(16 . R_{6}=H\right)$

- 1. $R_{1}, R_{2}, R_{3}, R_{4}=H, R_{5}=H A c, R_{6}=N_{3}\left(17, R_{6}=H\right)$

Scheme 2. (a) Nis-TfOH, $\mathrm{CH}_{2} \mathrm{Cl}_{2}, \mathrm{MS} 4 \AA, 0$ 'C, $5 \mathrm{~min}, 64 \%$; (b) $\mathrm{H}_{2}, 10 \% \mathrm{Pd} / \mathrm{C}, \mathrm{EtOH} / \mathrm{EtOAc}(2 / \mathrm{l}, \mathrm{v} / \mathrm{v}), \mathrm{rt}, 60 \mathrm{~h}$, (c) $\mathrm{Ac}_{2} \mathrm{O}, \mathrm{Py}, 1 \mathrm{tt}, 16$ h: (d) $\mathrm{NaN}_{3}, \mathrm{DMF}, 110^{\circ} \mathrm{C}$, it $4 \mathrm{~h}$ ( (e) 1 ) $\mathrm{N}_{2} \mathrm{H}_{4} \cdot \mathrm{H}_{2} \mathrm{O}, \mathrm{EtOH}, 70{ }^{\circ} \mathrm{C}$, lh, 2) $\mathrm{Ac}_{2} \mathrm{O}, \mathrm{Fy}, \mathrm{rt}, \mathrm{l} 6 \mathrm{~h}, 64 \%$ (from 8,5 steps); (f) NaOMe, $\mathrm{MeOH}, \mathrm{rt}, 3 \mathrm{~h}, 35 \%$ (from 8,6 steps). the carbon signals at 42.6 and 14.0 ppm show the presence of the chloroethyl glycoside 11 and ethyl glycoside 12 in a $5: 2$ ratio. This was also confirmed by the mass spectrum of the trisaccharides. The MALDI-TOF MS peaks of the produced trisaccharides showed at $\mathrm{m} / \mathrm{z}$ values of 1171.91 $(\mathrm{M}+\mathrm{Na})^{-}$for the chloroethyl glycoside 11 and at 1138 $\left(\mathrm{M}_{1}+\mathrm{Na}\right)^{+}$for the ethyl glycoside 12 . The sequential reactions of an azide displacement with $\mathrm{NaN}_{3}$ in DMF. a hydrazinolysis ${ }^{18.15}$ with $\mathrm{N}_{2} \mathrm{H}_{4} \mathrm{H}_{2} \mathrm{O}$ in EtOH and an acetylation transformed the mixture of 11 and 12 into the corresponding peracetylated mixture, 2 -azidoethyl $O-(2.3,4.7$-tetra- $O$ acetyl-6-deoxy- $\alpha$-D-alinoheptopy ranosyl)-(l $\rightarrow 3)$-(4.6-di- $O$ acetyl-2-deoxy -2- $N$-acety l- $\beta$-D-glucopyranosyl)-(l $\rightarrow 3$ )2.4,6-tri-O-acetyl- $\alpha$-D-galactopyranoside 15 and 2 -ethyl glycoside 16. The Zemplin de-O-acetylation ${ }^{2 i j}$ of the mixture 15 and 16 with $\mathrm{NaOMe}$ in $\mathrm{MeOH}$ gave the desired funal trisaccharide. 2'-azidoethyl O-(6-deosy- $\alpha$-D-altroheptopyranosyl $)-(\mathrm{l} \rightarrow 3)$-(2-deoxy-2-N-acetyl- $\beta$-D-glucopyranosyl)$(\mathrm{I} \rightarrow 3)-\alpha-\mathrm{D}$-galactopyranoside 1 and 2 -etlyyl glycoside 17 . as a white solid which was then purified using a Bio-gel P2 colunu. The overall yield after the six deprotection steps is in a $35 \%$. The MALDI-TOF MS spectrum of compound 1 (MW 628.6) showed a peak at $651.422(\mathrm{M}+\mathrm{Na})^{+}$. The presence of the side product $17,6 \mathrm{~d}$-altroHep-GlcNAc-Gal$\mathrm{OCH}_{2} \mathrm{CH}_{3}$ (MW 587.57), was also showed as an additional peak at $\mathrm{m} / \mathrm{z}$ value of $610.466(\mathrm{M}+\mathrm{Na})^{+}$. It is surprising that dechlorination took place during the hydrogenolysis of the trisaccharide $\mathbf{8}$, since it was not observed in the previous synthesis, i.e, the hydrogenolysis of another trisaccharide analogue, Gal-6d-altoHep-GlcNPhth- $O \mathrm{CH}_{2} \mathrm{CH}_{2} \mathrm{Cl}^{1{ }^{10}}$ Even the debenzylation was found to be much slower for $\mathbf{8}$ ( $6 \mathrm{~d}$ altroHep-GlcNPhth-Gal trisaccharide, $60 \mathrm{~h}$ ) than for the analogue (Gal-6d-altroHep-GlcNPhth trisaccharide, $26 \mathrm{~h}$ ). The longer reaction time for debenzylation and the concomitant dechlorination seems to ascribe to the steric environmental differences between the two trisaccharides. The 2'azidoethyl trisaccharide can be linked to a peptide (or a protein) for glyccoconjugation.

\section{Experimental}

General. ${ }^{1} \mathrm{H}$ and ${ }^{13} \mathrm{C}$ spectra were recorded on JEOL JNM-LA $400(400 \mathrm{MHz})$ using $\mathrm{CDCl}_{2}$ or $\mathrm{D}_{2} \mathrm{O}$ and chemical shifts are reported in parts per million $(\delta)$ downfield from tetramethylsilane as internal standard. Assiguments were based on DEPT, COSY, and HMQC. Matrix associated laser desorption time-of-flight (MALDI-TOF) spectra were recorded on Voyger Biospectrometry workstation with a $337 \mathrm{~nm}$ nitrogen laser and a $1.2 \mathrm{~m}$ linear mass analyzer (PerSeptive Bioșystems. Framingham. MA). TLC was performed on Merck pre-coated $60 \mathrm{~F}_{25+}$ plates. Columu chromatography was performed on silica gel (Merck. Art 9385 230-400 mesh in the flash mode). All the anhydrous solvents were distilled over $\mathrm{CaH}_{2}$ or $\mathrm{P}_{2} \mathrm{O}_{5}$ or $\mathrm{Na}$ /benzophenone prior to the reaction.

Ethylthio 3,4- $O$-isopropylidene- $\beta$-D-galactopyranoside (2). To a solution of ethylthio $\beta$-D-galactopyranoside $(0.6902$ g. $2.972 \mathrm{mmol}$ ) dissolved in THF (15 mL), $p$-toluenesulfonic 
acid $(0.0896$ g. $0.5201 \mathrm{mmol})$ and 2.2-dimethoxypropane $(0.73 \mathrm{~mL}, 5.944 \mathrm{mmol}$ ) were added and stirred for $2.5 \mathrm{hr}$ at room temperature. Triethylanine $(0.15 \mathrm{~mL})$ was added to the reaction mixture and evaporated to a syrup, which was chromatographed on silica gel (toluene-EtOAc. 5:3. followed by $1: 1$ and toluene-EtOAc-EtOH, 5:5:2) to give $2(0.5722$ g. $73 \%$ ) having $\mathrm{R}_{f} 0.56$ (toluene-EtOAc-EtOH, $5: 5: 2$ ). ${ }^{1} \mathrm{H}$ NMR $\left(\mathrm{CDCl}_{3}, 400 \mathrm{MHz}\right) \delta 4.30\left(\mathrm{~d}, J_{1.2}=10 \mathrm{~Hz}, \mathrm{lH}, \mathrm{H}-1\right)$. 4.21 (dd. $\left.J_{4.5}=1.96 \mathrm{~Hz} .1 \mathrm{H}, \mathrm{H}-4\right), 4.10\left(\mathrm{t}, J_{3.4}=5.60 \mathrm{~Hz}, \mathrm{lH}\right.$. $\mathrm{H}-3), 3.97-3.79$ (n. $3 \mathrm{H}, \mathrm{H}-6 \mathrm{a} . \mathrm{H}-5, \& \mathrm{H}-6 \mathrm{~b}), 3.56$ (d. $J_{3.3}=$ $7.32 \mathrm{~Hz} . \mathrm{lH}, \mathrm{H}-2$ ), 3.32 (s. $1 \mathrm{H}, \mathrm{OH}$ ), 2.94 (s. $1 \mathrm{H}, \mathrm{OH}$ ) $2.80-$ 2.71 (m. $2 \mathrm{H}$. SCH 2 ). 1.52 (s. $3 \mathrm{H}_{\mathrm{SC}} \mathrm{SCH}_{2} \mathrm{CH}_{3}$ ), $1.35-1.30$ (m. $\left.6 \mathrm{H}, \mathrm{C}\left(\mathrm{CH}_{3}\right)=\right) ;{ }^{13} \mathrm{C}-\mathrm{NMR}\left(\mathrm{CDCl}_{3}, 100 \mathrm{MHz}\right) \delta 110.0(\underline{\mathrm{C}}$ $\left(\mathrm{CH}_{3}\right)=85.0(\mathrm{C}-1) .79 .0(\mathrm{C}-3) .76 .8(\mathrm{C}-5) .73 .6(\mathrm{C}-4) .71 .8$ $(\mathrm{C}-2) .62 .0(\mathrm{C}-6), 27.9 \& 26.1\left(\mathrm{C}\left(\mathrm{CH}_{3}\right)_{2}\right), 24.1\left(\mathrm{~S}_{-} \mathrm{H}_{5}\right), 15.0$ $\left(\mathrm{SCH}_{2} \mathrm{CH}_{3}\right)$

Ethylthio 2,6-di- $O$-benzyl-3,4-O-isopropylidene- $\beta$-Dgalactopyranoside (3). Compound $2(0.728$ g. 0.0275 mmol) in DMF (21 mL) was cooled at $0{ }^{\circ} \mathrm{C}$ and $\mathrm{NaH}(60 \%$ in mineral oil. $0.7933 \mathrm{~g}$ ) was added and stirred for $40 \mathrm{~min}$. Then benzyl bronide $(1.64 \mathrm{~mL} .0 .1377 \mathrm{mmol})$ was added dropwise at $0^{\circ} \mathrm{C}$ and stirred for $\mathrm{l}$ hr at room temperature and then concentrated. The residual syrup was diluted with $\mathrm{CH}_{2} \mathrm{Cl}_{2}$, washed with water, dried and concentrated to a syrup, which was chromatographyed on silica gel (tolueneEtOAc. 50:1) to give $3\left(1.22+\right.$ g. quantitative) having $\mathrm{R}_{f}$ 0.38 (toluene-EtOAc, 15:I). ${ }^{1} \mathrm{H}-\mathrm{NMR}\left(\mathrm{CDCl}_{3} .400 \mathrm{MHz}\right) \delta$ 7.41-7.21 (m. 10H, aromatic $\mathrm{H}$ ). 4.84-4.47 (m. $4 \mathrm{H}^{\mathrm{C}} \mathrm{C}_{6} \mathrm{H}_{5} \mathrm{C}_{2}$ ), $4.40\left(\mathrm{~d}, J_{1.2}=11 \mathrm{~Hz} .1 \mathrm{H}, \mathrm{H}-1\right) .4 .16-4.12(\mathrm{~m} .2 \mathrm{H}, \mathrm{H}-4 \& \mathrm{H}-$ 3), 3.86-3.83 (m. 1H, H-5), 3.74-3.73 (m, 2H. H-6). 3.463.42 (dd. $\left.J_{2.3}=6.36 \mathrm{~Hz} . \mathrm{HH} . \mathrm{H}-2\right) .2 .64$ (m. $2 \mathrm{H} . \mathrm{SCH}$ ), 1.40 (s. $\left.3 \mathrm{H}, \mathrm{SCH}_{2} \mathrm{CH}_{3}\right) .1 .31-1.26$ (m. $\left.6 \mathrm{H} . \mathrm{C}\left(\mathrm{CH}_{3}\right)_{2}\right) ;{ }^{13} \mathrm{C}-\mathrm{NMR}$ $\left(\mathrm{CDCl}_{3}, 100 \mathrm{MHz}\right) \delta 138.1-127.3$ (aromatic C). $109.6(\mathrm{C}$ $\left(\mathrm{CH}_{3}\right)=83.5(\mathrm{C}-1) .79 .4(\mathrm{C}-3) .78 .9(\mathrm{C}-2) .75 .4(\mathrm{C}-5) .73 .7$ (C.4). $71.8\left(\mathrm{C}_{6} \mathrm{H}_{5} \mathrm{CH}_{2}^{*} 2\right), 69.4(\mathrm{C}-6), 27.7 \& 26.1(\mathrm{C}$ $\left.\left(\mathrm{CH}_{3}\right)_{2}\right), 24.4\left(\mathrm{~S}^{2} \mathrm{CH}_{2}\right) .14 .8\left(\mathrm{SCH}_{2} \mathrm{CH}_{3}\right)$.

2'-Chloroethyl 2,6-di-O-benzyl-3,4-O-isopropylidene$\alpha$-D-galactopyranoside (t) \& 2'-chloroethyl 2,6-di- $O$ benzyl-3,4- $O$-isopropylidene- $\beta$-D-galactopyranoside (5). i) Employing IDCP promoter. A solution of $3(0.3499 \mathrm{~g}$. $0.787 \mathrm{mmol}$ ) and 2-chloroethanol $(53 \mu \mathrm{L} .0 .7873 \mathrm{mmol})$ in $\mathrm{CH}_{2} \mathrm{Cl}_{2}-\mathrm{Et}_{2} \mathrm{O}(2.5 \mathrm{r} / \mathrm{v} .28 \mathrm{~mL})$ was stirred with freshly powdered MS $5 \AA \mathrm{A}(2 \mathrm{~g})$ for $30 \mathrm{~min}$ at room temperature and then iodonium dicollidine perchlorate (IDCP; 1.105 g. 2.361 $\mathrm{mmol}$ ) was added. After stirring $30 \mathrm{~min}$ at room temperature. the precipitate was filtered off through celite-bed. and washed with $\mathrm{CH}_{2} \mathrm{Cl}_{\text {. }}$. The combined filtrate was washed with $1 \mathrm{M}$ $\mathrm{Na}_{2} \mathrm{~S}_{3} \mathrm{O}_{3}$ and water. dried and then concentrated. Colunn chromatography (toluene-EtOAc. 15:1) of the residue gave $\alpha,+(0.1976 \mathrm{~g} .54 \%)$ and $\beta .5(0.0387 \mathrm{~g} .11 \%)$ having $\mathrm{R}_{f}$ 0.59 for $t$ and 0.52 for 5 (toluene-EtOAc. $5: 1$ ).

ii) Employing NIS-TfOH promoter. A solution of 3 (7.52 g. $0.0163 \mathrm{~mol}$ ) and 2 -chloroethanol $(1.65 \mathrm{~mL} .0 .0245 \mathrm{~mol})$ in $\mathrm{CH}_{2} \mathrm{Cl}_{2}(72 \mathrm{~mL})$ was stirred with freshly powdered MS 4 $(2 \mathrm{~g})$ for $30 \mathrm{~min}$ at room temperature and then cooled to 0 ${ }^{\circ} \mathrm{C}$. To the cooled mixture was added. with stirring, $\mathrm{N}$-iodosuccinimide (NIS: $9.173 \mathrm{l}$ g. $0.0408 \mathrm{~mol}$ ) and trifluoro- methane-sulfonic acid (TfOH; $453 \mu \mathrm{L}$. $0.0051 \mathrm{mmol}$ )/ $\mathrm{CH}_{2} \mathrm{Cl}_{2}(5 \mathrm{~mL})$. The reaction mixture was stirred for $10 \mathrm{~min}$ at $0^{\circ} \mathrm{C}$. The precipitate was filtered through celite-bed. and washed with $\mathrm{CH}_{2} \mathrm{Cl}_{2}$. The combined filtrate was washed with $1 \mathrm{M} \mathrm{Na}_{2} \mathrm{~S}_{2} \mathrm{O}_{3}$. water. $\mathrm{NaHCO}_{3}$, and water. dried and then concentrated. which was separated on silica gel (tolueneEtOAc, $15: 1)$ to give $\alpha \mathbf{4}(4.486 \mathrm{~g} .59 \%)$ and $\beta .5(2.597 \mathrm{~g}$. $34 \%$ ). ${ }^{1} \mathrm{H}-\mathrm{NMR}\left(\mathrm{CDCl}_{3} .400 \mathrm{MHz}\right)$ for $+\delta 7.38-7.24(\mathrm{~m}$, $10 \mathrm{H}$, aromatic $\mathrm{H}), 4.83-4.52\left(\mathrm{~m}, 4 \mathrm{H} . \mathrm{C}_{6} \mathrm{H}_{5} \mathrm{C}_{2}{ }^{*} 2\right), 4.82(\mathrm{~d}$. $\left.J_{1.2}=3.4 \mathrm{~Hz} . \mathrm{H} . \mathrm{H}-1\right), 4.35\left(\mathrm{dd}, J_{3.4}=5.6 \mathrm{~Hz}, 1 \mathrm{H}, \mathrm{H}-3\right)$, $4.32-4.28$ (m, IH. H-5), 4.20 (dd. $J_{4.5}=2.44 \mathrm{~Hz}, 1 \mathrm{H}, \mathrm{H}-4$ ), $3.89-3.66$ (m, $6 \mathrm{H} . \mathrm{OCH}_{2} \mathrm{CH}_{2} \mathrm{Cl}, \mathrm{CH}_{2} \mathrm{Cl} . \mathrm{H}-6$ ), 3.53 (dd. $J_{2.3}$ $=7.58 \mathrm{~Hz} .1 \mathrm{H} . \mathrm{H}-2)$. $1.39 \& 1.33$ (each s, $\left.6 \mathrm{H}, \mathrm{C}\left(\mathrm{CH}_{3}\right)_{2}\right)$ : ${ }^{13} \mathrm{C}-\mathrm{NMR}\left(\mathrm{CDCl}_{3}, 100 \mathrm{MHz}\right)$ for $+\delta 138.2-127.5$ (aromatic C). $109.2\left(\mathrm{C}\left(\mathrm{CH}_{3}\right)_{2}\right) .97 .4(\mathrm{C}-1) .76 .3(\mathrm{C}-2) .75 .6(\mathrm{C}-3) .73 .6$ $(\mathrm{C}-4), 73.3 \& 72.5\left(\mathrm{C}_{6} \mathrm{H}_{5} \mathrm{CH}_{2} * 2\right) .69 .4(\mathrm{C}-6) .68 .4\left(\mathrm{OCH}_{2}-\right.$ $\left.\left.\mathrm{CH}_{2} \mathrm{Cl}\right) .66 .9(\mathrm{C}-5) .42 .6\left(\mathrm{CH}_{2} \mathrm{Cl}\right), 28.0 \& 26.3\left(\mathrm{C}_{(\mathrm{C}}\right)_{3}\right)$ ): ${ }^{1} \mathrm{H}-\mathrm{NMR}\left(\mathrm{CDCl}_{3}, 400 \mathrm{MHz}\right)$ for $5 \delta 7.41-7.25(\mathrm{~m} . \mathrm{lOH}$, aromatic $\mathrm{H}), 4.88-4.53\left(\mathrm{~m}, 4 \mathrm{H}, \mathrm{C}_{\mathrm{i}} \mathrm{H}_{2} \mathrm{CH}_{2} * 2\right) .4 .36\left(\mathrm{~d} . J_{1.2}=\right.$ $8.04 \mathrm{~Hz}, \mathrm{lH}, \mathrm{H}-\mathrm{l}), 4.13(\mathrm{~m} .3 \mathrm{H}) .3 .9 \mathrm{l}(\mathrm{t}, \mathrm{lH}), 3.83-3.67(\mathrm{~m}$. $5 \mathrm{H}$. $\mathrm{OC}_{2} \mathrm{CH}_{2} \mathrm{Cl}$ C $\underline{\mathrm{H}}_{2} \mathrm{Cl}$ ), 3.40 (dd. $J_{2,3}=6.36 \mathrm{~Hz}, \mathrm{lH}, \mathrm{H}-$ 2), $1.35 \& 1.32$ (each $\left.\mathrm{s}, 6 \mathrm{H}, \mathrm{C}\left(\mathrm{CH}_{3}\right)_{2}\right):{ }^{13} \mathrm{C}-\mathrm{NMR}\left(\mathrm{CDCl}_{3}\right.$, $100 \mathrm{MHz}$ ) for $5 \delta 138.2-127.5$ (aromatic $\mathrm{C}$ ). $109.9\left(\mathrm{C}\left(\mathrm{CH}_{3}\right)_{2}\right)$, 103.1 (C-1), 79.2. 78.8. 73.7, 73.6\& $73.5\left(\mathrm{C}_{6} \mathrm{H}_{5} \underline{\mathrm{C}} \mathrm{H}_{2} * 2\right)$, $72.2,69.6\left(\mathrm{OCH}_{2} \mathrm{CH}_{2} \mathrm{Cl}\right), 69.5(\mathrm{C} \cdot 6), 42.6\left(\mathrm{CH}_{2} \mathrm{Cl}\right), 27.7 \&$ $26.3\left(\mathrm{C}\left(\mathrm{CH}_{3}\right)_{2}\right)$.

2'-Chloroethyl 2,6-di- $O$-benzyl- $\alpha$-D-galactopyranoside (6). To a solution of $+(2.414 \mathrm{~g} .5 .214 \mathrm{mmol})$ in $\mathrm{MeOH}(100$ $\mathrm{mL})$ was added $p$-toluenesulfonic acid $(0.1984 \mathrm{~g} .1 .043$ munol) with stirring for $40 \mathrm{~min}$ at $65^{\circ} \mathrm{C}$. Trietlyylamine $(0.3$ $\mathrm{ml}$ ) was added to the reaction mixture and evaporated to dryness. Column cluromatography (toluene-EtOAc. 5:3) of the residue gave $6\left(2.035 \mathrm{~g} .91 \%\right.$ ) having $\mathrm{R}_{f} 0.30$ (tolueneEtOAc, 53). ${ }^{\mathrm{H}} \mathrm{H}-\mathrm{NMR}(\mathrm{CDCl}, 400 \mathrm{MHz}) \delta 7.38-7.26(\mathrm{~m}$, $10 \mathrm{H}$, aromatic H), 4.85 (d. $\left.J_{1.2}=3.68 \mathrm{~Hz} .1 \mathrm{H} . \mathrm{H}-1\right) .4 .71-$ $4.56\left(\mathrm{~m}, 4 \mathrm{H}, \mathrm{C}_{6} \mathrm{H}_{5} \mathrm{CH}_{2} * 2\right) .4 .04-3.97(\mathrm{~m}, 3 \mathrm{H}, \mathrm{H}-4 . \mathrm{H}-5.82$ $\mathrm{H}-3), 3.85-3.63$ (m. $7 \mathrm{H}, \mathrm{OC}_{2} \mathrm{CH}_{2} \mathrm{Cl}$. $\mathrm{CH}_{2} \mathrm{Cl} . \mathrm{H}-6, \& \mathrm{H}-2$ ), $3.00(\mathrm{~d} . J=1.24 \mathrm{~Hz} .1 \mathrm{H} . \mathrm{OH}) .2 .77(\mathrm{~d} . J=3.64 \mathrm{~Hz} .1 \mathrm{H}$. $\mathrm{OH}):{ }^{13} \mathrm{C}-\mathrm{NMR}\left(\mathrm{CDCl}_{3}, 100 \mathrm{MHz}\right) \delta 138.0-127.6$ (aromatic C) 97.2 (C-1). $76.6(\mathrm{C}-2), 73.5 \& 72.8\left(\mathrm{C}_{6} \mathrm{H}_{2} \mathrm{CH}_{2} * 2\right), 69.8$ (C-6). 69.7 (C-4), 69.1 (C-3). 68.8 (C-5), $68.3\left(\mathrm{OCH}_{2} \mathrm{CH}_{2} \mathrm{Cl}\right)$, $42.8\left(\mathrm{CH}_{2} \mathrm{Cl}\right)$.

2'-Chloroethyl $O$-(3-O-acetyl-7-O-benzoyl-2,4-di- $O$-benzyl6-deoxy- $\alpha$-D-attroheptopyranosyl)-(1 $\rightarrow 3)-(4,6-$ di $-O$-acetyl2-deoxy-2-phthalimido- $\beta$-D-glucopyranosyl)-( $(1 \rightarrow 3)$-2,6di- $O$-benzyl- $\alpha$-D-galactopyranoside (8). A solution of ethyl $O$-(3-O-acetyl-7-O-benzoyl-2.4-di-O-benzyl-6-deosy$\alpha$-D-altroheptopy ranosyl)-(13)-4,6-di-O-acetyl-2-deoxy-2phthalimido-1-thio- $\beta$-D-glucopyranoside $7^{1 \mathrm{li}}(0.22 \mathrm{~g} .0 .234$ nmol) and $6(0.2017 \mathrm{~g} .0 .468 \mathrm{mmol})$ in $\mathrm{CH}_{2} \mathrm{Cl}_{2}(10 \mathrm{~mL})$ was stirred with freshly powdered MS $4(\mathrm{lg})$ for $30 \mathrm{~min}$ at room temperature and then cooled to $0^{\circ} \mathrm{C}$. To the cooled mixture were added. with stirring. $N$-iodosuccinimide (NIS; 0.1316 g. $0.585 \mathrm{mmol}$ ) and trifluoromethanesulfonic acid (TfOH: $6.6 \mu \mathrm{L}$. $0.0748 \mathrm{mmol} / \mathrm{CH}_{2} \mathrm{Cl}_{2}(0.6 \mathrm{~mL})$. The reaction mixture was stirred for $5 \mathrm{~min}$ at $0{ }^{\circ} \mathrm{C}$. The precipitate was filtered through celite-bed. and washed with $\mathrm{CH}_{2} \mathrm{Cl}_{2}$. The 
combined filtrate was washed with $1 \mathrm{M} \mathrm{Na} \mathrm{S}_{2} \mathrm{O}_{3}$. water. $\mathrm{NaHCO}_{3}$, and water, dried and then concentrated. which was chromato-graphed on silica gel (toluene-EtOAc. $4: 1$ ) to give $8(0.196$ g. $64 \%)$ having $\mathrm{R}_{f} 0.55$ (toluene-EtOAc, 5:3, v/v). ${ }^{1} \mathrm{H}-\mathrm{NMR}\left(\mathrm{CDCl}_{3} .400 \mathrm{MHz}\right)$ for $7 \delta 7.95-6.97(\mathrm{~m}, 19 \mathrm{H}$. aromatic H). $5.29\left(\mathrm{~d}, J_{1.2}=10.48 \mathrm{~Hz}, \mathrm{lH} . \mathrm{H}-\mathrm{l}\right), 5.05\left(\mathrm{t}, J_{4.5}=\right.$ $9.64 \mathrm{~Hz} .1 \mathrm{H}, \mathrm{H}-4), 4.95$ (dd. $\left.J_{3^{\prime} .4^{\prime}}=2.72 \mathrm{~Hz}, 1 \mathrm{H}, \mathrm{H}-3^{\prime}\right), 4.58-$ 3.96 (m. 9H, H-l', $\mathrm{C}_{6} \mathrm{H}_{5} \mathrm{CH}_{2} * 2, \mathrm{H}-7$ ' \& H-6). 4.49 (t. $J_{3.4}=$ $9.64 \mathrm{~Hz}$. IH. H-3), 4.28 (t. $J_{2.3}=10.28 \mathrm{~Hz}$. IH. H-2), $3.67-$ $3.62(\mathrm{~m}, 1 \mathrm{H}, \mathrm{H}-5), 3.48\left(\mathrm{dd}, J_{2.3^{\prime}}=6.7 \mathrm{~Hz} .1 \mathrm{H}, \mathrm{H}-2^{\prime}\right), 3.38-$ $3.33\left(\mathrm{~m}, 2 \mathrm{H}, \mathrm{H}-5^{\prime} \& \mathrm{H}-4^{\prime}\right), 2.66-2.54\left(\mathrm{~m}, 2 \mathrm{H}, \mathrm{SCH}_{2}\right), 2.0 \mathrm{l}$. $1.86 \& 1.76$ (each s. $\left.9 \mathrm{H} . \mathrm{CH}_{3} * 3\right) .1 .76\left(\mathrm{~m}, \mathrm{lH} . \mathrm{H}-6 \mathrm{a}^{\prime}\right), 1.51-$ $1.46\left(\mathrm{~m}, 1 \mathrm{H} . \mathrm{H}-6 \mathrm{~b}^{\prime}\right) .1 .13$ (t, $\left.3 \mathrm{H}, \mathrm{SCH}_{2} \mathrm{CH}_{3}\right):{ }^{13} \mathrm{C}-\mathrm{NMR}$ $\left(\mathrm{CDCl}_{3}, 100 \mathrm{MHz}\right)$ for $7 \delta 170.62,170.59 \& 169.4(\mathrm{C}=\mathrm{O}$. Ac), 168.4\&167.2 (C=O. NPhth), 166.1 ( $\mathrm{C}=\mathrm{O}, \mathrm{Bz}) .137 .9$ 122.9 (aromatic C), 100.5 (C-1'), $81.3(\mathrm{C}-1), 77.5(\mathrm{C}-3) .76 .4$ (C-2'), $76.0(\mathrm{C}-5), 73.5\left(\mathrm{C}-4^{\prime}\right), 73.7 \& 70.5\left(\mathrm{C}_{6} \mathrm{H}_{3} \mathrm{CH}_{2} * 2\right)$. 70.9 (C-4), 68.2 (C-3'\& C-5'), $62.5(\mathrm{C}-6), 61.2\left(\mathrm{C}-7^{\prime}\right), 54.5$ (C.2). 28.8 (C-6'). 24.1 ( $\left.\mathrm{SCH}_{-}\right), 20.7 \& 20.4\left(\mathrm{CH}_{3} * 3\right) .14 .8$ $\left(\mathrm{SCH}_{2} \mathrm{CH}_{3}\right) ;{ }^{1} \mathrm{H}-\mathrm{NMR}\left(\mathrm{CDCl}_{3} .400 \mathrm{MHz}\right)$ for $8 \delta 7.94-6.95$ (m, $29 \mathrm{H}$, aromatic $\mathrm{H}$ ), $5.38\left(\mathrm{~d}, J_{1} \cdot 2=8.52 \mathrm{~Hz} \cdot 1 \mathrm{H}, \mathrm{H}-\mathrm{l}\right.$ '). $5.01\left(\mathrm{t}, J_{4.5}=9.52 \mathrm{~Hz} . \mathrm{lH}, \mathrm{H}-4^{\prime}\right) .4 .96\left(\mathrm{~d}, J_{3^{\prime \prime}} 4^{\prime \prime}=5.36 \mathrm{~Hz}\right.$. lH. H-3"), 4.57-3.93 (m, 20H. H-l". H-3'. $\mathrm{C}_{6} \mathrm{H}_{5} \mathrm{CH}_{2}{ }^{*} 4, \mathrm{H}-\mathrm{l}$. $\mathrm{H}-2^{\prime}$. H-4. OCH $\left.\mathrm{H}_{2} \mathrm{CH}_{2} \mathrm{Cl}, \mathrm{H}-5, \mathrm{H}-7^{\prime \prime} \& \mathrm{H}-6^{\prime}\right) .3 .88$ (dd. $J_{3.4}=$ $3.16 \mathrm{~Hz} .1 \mathrm{H}, \mathrm{H}-3), 3.68-3.46$ (m, 7H. H-5', H-2. $\mathrm{H}-6, \mathrm{CH}_{2} \mathrm{Cl}$ \& H-2"). 3.38 (s. $2 \mathrm{H}, \mathrm{H}-5^{\prime \prime} \& \mathrm{H}-4 "$ ). 2.62 (s, $1 \mathrm{H}, 4-\mathrm{OH}$ ). $1.92,1.88 \& 1.77$ (each s, 9H, $\mathrm{CH}_{3}^{*} 3$ ). 1.77 (m. 1H. H-6a"). $1.46(\mathrm{~m}, \mathrm{lH} . \mathrm{H}-6 \mathrm{~b})$ ); ${ }^{13} \mathrm{C}-\mathrm{NMR}\left(\mathrm{CDCl}_{3}, 100 \mathrm{MHz}\right)$ for $8 \delta$ 170.7. $170.6 \& 169.5(\mathrm{C}=\mathrm{O}$. Ac) $168.5 \& 167.5(\mathrm{C}=\mathrm{O}$. NPhth). $166.2(\mathrm{C}=\mathrm{O} . \mathrm{Bz}$ ). 138.3-123.2 (aromatic C). 100.6 (C-1"), 98.9 (C-1'). 98.0 (C-1), 79.8 (C-3), $76.7\left(\mathrm{C}-3^{\prime}\right), 76.3$ (C-2"). $74.4(\mathrm{C}-2) .73 .4$ (C-4"). 73.6, 73.4. $73.3 \& 70.5$ $\left(\mathrm{C}_{6} \mathrm{H}_{5} \mathrm{CH}_{2}^{*} 4\right) .71 .9\left(\mathrm{C}-5^{\prime}\right), 70.9\left(\mathrm{C}-4^{\prime}\right), 69.4(\mathrm{C}-6), 68.9(\mathrm{C}-$ 4), $68.4(\mathrm{C}-5) .68 .3\left(\mathrm{CH}_{2} \mathrm{CH}_{2} \mathrm{Cl}\right), 68.0\left(\mathrm{C}-3^{\prime \prime} \& \mathrm{C}-5^{\prime \prime}\right) .62 .3$ (C.6'). $61.1\left(\mathrm{C}^{\prime}-7^{\prime \prime}\right) .55 .7\left(\mathrm{C}-2^{\prime}\right), 42.5\left(\mathrm{CH}_{2} \mathrm{Cl}\right) .28 .9\left(\mathrm{C}-6^{\prime \prime}\right)$. $20.8,20.6 \& 20.5\left(\mathrm{CH}_{3}^{*} 3\right)$.

2'-Azidoethyl $O$-(2,3,4,7-tetra- $O$-acetyl-6-deoxy- $c-D$ - $a$ ltroheptopyranosyl)-(1 $\rightarrow 3)$-(4,6-di-O-acetyl-2-deoxy-2- $N$ acetyl- $\beta$-D-glucopyranosyl)-(1 $\rightarrow 3)-2,4,6-$ tri- $O$-acetyl- $\alpha$ D-galactopyranoside (15) \& 2'-ethyl $O-(2,3,4,7$-tetra- $O$ acetyl-6-deoxy- $\alpha$-D-altroheptopyranosyl)-(1 $\rightarrow 3)-(+, 6$-di$O$-acetyl-2-deoxy-2- $N$-acetyl- $\beta$-D-glucopyranosyl)-(1 $\rightarrow 3$ ) -2,4,6-tri- $O$-acetyl- $\alpha-\mathrm{D}$-galactopyranoside (16). Compound $8(0.0846 \mathrm{~g}, 0.0646 \mathrm{mmol})$ in EtOAc-EtOH $(1: 2,12 \mathrm{~mL})$ was hydrogenated in the presence of $10 \% \mathrm{Pd} / \mathrm{C}$ for $60 \mathrm{hr}$ at room temperature. The reaction mixture was filtered over celite-bed and concentrated to dryness. The residue was dissolved in pyridine $(5 \mathrm{~mL})$ and treated with acetic anhydride $(5 \mathrm{~mL})$. After stirring for $16 \mathrm{hr}$ at room temperature. the mixture was concentrated. The synupy mixture $(11 \& 12)$ was dissolved in DMF $(5 \mathrm{~mL})$ stirred with sodium azide $(0.0063 \mathrm{~g} .0 .0967 \mathrm{mmol})$ at $110^{\circ} \mathrm{C}$ for $4 \mathrm{hr}$, the mixture was cooled, evaporated and then diluted in $\mathrm{CH}_{2} \mathrm{Cl}_{\underline{2}}$. The organic layer was washed with water. dried. and concentrated. Without further purification. the residue was dissolved in $\mathrm{EtOH}(5 \mathrm{~mL})$ and treated with hydrazine monohydrate $(98 \% .3 \mathrm{~mL})$. After stirring for 1 lor at $72^{\circ} \mathrm{C}$, the mixture was coevaporated with toluene. Acetic anhydride $(7 \mathrm{~mL})$ was added and then dried after stirring for $16 \mathrm{hr}$ at room temperature. Column cluromatography (toluene-EtOAc-EtOH, 5:5:1) of the residue gave mixture 15 and $16(0.0414$ g. $64 \%$ from 8. 5 steps) having $\mathrm{R}_{f} 0.48$ (toluene-EtOAc-EtOH. 5:5:2, $\mathrm{v} / \mathrm{v} /$ v). ${ }^{\mathrm{H}} \mathrm{H}-\mathrm{NMR}\left(\mathrm{CDCl}_{3} .400 \mathrm{MHz}\right.$ ) for the mixture of $\mathbf{1 1}$ and 12 $\delta 7.94-7.11(\mathrm{~m}, 9 \mathrm{H}$, aromatic $\mathrm{H}) .5 .36\left(\mathrm{dd} . J_{4.5}=7.66 \mathrm{~Hz}\right.$, 1H. H-4). $5.23\left(\mathrm{~d}, J_{\mathrm{l}^{\prime} .2}=8.52 \mathrm{~Hz}\right.$. lH, H-1'), $5.05\left(\mathrm{t}, J_{4^{\prime}, 5^{\prime}}=\right.$ $\left.8.78 \mathrm{~Hz}, \mathrm{lH}, \mathrm{H}-4^{\prime}\right) .4 .96\left(\mathrm{~m}, \mathrm{lH}, \mathrm{H}-3^{\prime \prime}\right), 4.87$ (d. $J_{1.2}=3.65$ Hz. $1 \mathrm{H}, \mathrm{H}-1$ ). 4.80 (dd. $J_{4^{\prime \prime .5 " ~}}=7.2 \mathrm{~Hz} . J_{3^{\prime \prime}} .^{\prime \prime}=3.44 \mathrm{~Hz}, \mathrm{lH}$, $\mathrm{H}-4$ "). 4.73-4.69 (m. 2H, H-2" \& H-2), 4.54 (m, lH, H-l"), 4.49 (t. $\left.J_{3^{\prime}, 4^{\prime}}=9.76 \mathrm{~Hz}, \mathrm{lH} . \mathrm{H}-3\right) .4 .20-3.54\left(\mathrm{~m}, 15 \mathrm{H}, \mathrm{H}-6^{\prime}\right.$, H-2', H-3. H-5, H-6. H-7". H-5'. H-5". OCH $\& \mathrm{CH}_{2} \mathrm{Cl}$ ), 2.06. 2.05. 2.03, 2.02. 2.00. $1.99,1.98 \& 1.97$ (each s. $24 \mathrm{H}$, $\mathrm{CH}_{3}{ }^{* 8)}$ ). 1.57 (m, lH, H-6a"). 1.43 (m, lH. H-6b"), 1.11 (t, 1H. $\left.\mathrm{CH}_{2} \mathrm{CH}_{3}\right) ;{ }^{13} \mathrm{C}-\mathrm{NMR}\left(\mathrm{CDCl}_{3}, 100 \mathrm{MHz}\right)$ for the mixture of 11 and $12 \delta 170.8,170.5 .170 .46 .170 .3 .169 .9 .169 .6$. 169.4, \& 169.2 ( $\mathrm{C}=\mathrm{O} * 8, \mathrm{Ac}) .168 .6 \& 167.0(\mathrm{C}=\mathrm{O}$, NPhth $)$. 166. I (C=O. Bz). 134.0-122.8 (aromatic C), $99.2 \& 99.1$ (Cl"). $98.1 \& 98.0(\mathrm{C}-1$ ') $96.1 \& 95.5$ (C-1). 78.1 (C-3'). 71.9 (C-5'). $71.6(\mathrm{C}-3), 70.4$ (C-4), 70.2 (C-2). $69.8(\mathrm{C}-4) .69 .1$ $\left(\mathrm{C}-2^{\prime \prime}\right) .68 .5\left(\mathrm{OCH}_{2}\right), 68.0\left(\mathrm{C}-4^{\prime \prime}\right), 67.2\left(\mathrm{C}-3^{\prime \prime}\right), 67.0\left(\mathrm{C}-5^{\prime \prime}\right)$, 66.7 (C-5). $62.5(\mathrm{C}-6), 61.7\left(\mathrm{C}-6^{\prime}\right), 60.3\left(\mathrm{C}-7^{\prime \prime}\right) .55 .8\left(\mathrm{C}-2^{\prime}\right)$, $42.6\left(\mathrm{CH}_{2} \mathrm{Cl}\right) .29 .6(\mathrm{C}-6 "), 20.74,20.7 .20 .64,20.61,20.55$, 20.51. 20.5. 20.3 $\left(\mathrm{CH}_{3}, \mathrm{Ac}\right), 14.0\left(\mathrm{CH}_{2} \mathrm{CH}_{3}\right)$ : MALDI-TOF MS $1171.91 \& 1138\left[(\mathrm{M}+\mathrm{Na}) \&\left(\mathrm{M}_{1}+\mathrm{Na}\right)\right]:{ }^{13} \mathrm{C}-\mathrm{NMR}$ $\left(\mathrm{CDCl}_{3} .100 \mathrm{MHz}\right)$ for the mixture of $\mathbf{1 5}$ and $\mathbf{1 6} \delta 172.3$, $171.8,171.7,171.4 .171 .2 .171 .1,170.8 .170 .5 .170 .3 \&$ $170.0\left(\mathrm{C}=\mathrm{O}^{*} 10 . \mathrm{Ac} \& \mathrm{NHAc}\right), 100.2 \& 100.0\left(\mathrm{C}-\mathrm{l}^{\prime}\right), 99.39$ $\& 99.35$ (C-1"). $96.6 \& 96.1$ (C-1), 79.0, 72.7, 71.8, 71.0, 70.8. 70.0, 69.6, 68.6. 67.6, 67.57 ( $\left.\mathrm{OCH}_{2}\right) .67 .1,66.3 .63 .0$, $62.3,60.5 .57 .5\left(\mathrm{C}-2^{\prime}\right) .50 .7\left(\mathrm{CH}_{2} \mathrm{~N}_{3}\right) .32 .3\left(\mathrm{C}-6^{\prime \prime}\right), 26.5$ $\left(\mathrm{CH}_{3} \mathrm{NHAc}\right), 21.1$. 20.92. 20.9, 20.86. 20.84. 20.8, 20.75. $20.7 \& 20.6\left(\mathrm{CH}_{3} * 9, \mathrm{Ac}\right) .15 .0\left(\mathrm{CH}_{2} \mathrm{CH}_{3}\right) ; \mathrm{IR} 2110.26 \mathrm{~cm}^{-\mathrm{i}}$ $\left(\mathrm{N}_{3}\right) .1739 .90(\mathrm{C}=\mathrm{O})$; MALDI-TOF MS $1029.25 \& 988.242$ $\left[(\mathrm{M}+\mathrm{Na}) \&\left(\mathrm{M}_{1}+\mathrm{Na}\right)\right]$.

2'-Azidoethyl $O$-(6-deoxy- $\alpha$-D-altroheptopyranosyl)(13)-(2-deoxy-2- $N$-acetyl- $\beta$-D-glucopyranosyl)-(1 $\rightarrow 3$ )- $\alpha$ D-galactopyranoside (1) \& 2'-ethyl $O$-(6-deoxy- $\alpha-D$ altroheptopyranosyl)-(1 $\rightarrow 3)$-(2-deoxy-2- $N$-acetyl- $\beta$-Dglucopyranosyl)-(1 $\rightarrow$ 3)- $\alpha$-D-galactopyranoside (17). A mixture of compound 15 and $16(0.0192 \mathrm{~g} .0 .019 \mathrm{mmol})$ was treated with $25 \% \mathrm{NaOMe}(0.01 \mathrm{~mL})$ in $\mathrm{MeOH}(1 \mathrm{~mL})$ for 3 $\mathrm{hr}$ at room temperature. The reaction mixture was neutralized with Dowex $50\left(\mathrm{H}^{+}\right)$resin, filtered, and concentrated to a solid $(1 \& 17)$. The crude product was treated to Bio-gel P2 column to give a mixture 1 and $17(0.0042 \mathrm{~g} .35 \%$ from 8.6 steps) having $\mathrm{R}_{f} 0.07\left(t \mathrm{BuOH}-\mathrm{EtOAc}-\mathrm{AcOH}-\mathrm{H}_{2} \mathrm{O} .36: 36: 7\right.$ : 21). ${ }^{13} \mathrm{C}-\mathrm{NMR}\left(\mathrm{D}_{2} \mathrm{O} .100 \mathrm{MHz}\right)$ for 1 and $17 \delta 177.8(\mathrm{C}=\mathrm{O})$, 105.2 (C-1"), 102.9 (C-1'), 101.4 (C-1). 82.5, 82.0, 78.3.73.6, 73.24. 73.2. 73.0. 72.8.71.9. 71.4. 70.0. 69.4 ( $\left.\mathrm{OCH}_{2}\right) .64 .0$, $63.1 \& 60.8\left(\mathrm{C}-6, \mathrm{C}-6^{\prime} \& \mathrm{C}-7^{\prime \prime}\right) .57 .1\left(\mathrm{C}-2^{\prime}\right), 53.3\left(\mathrm{CH}_{2} \mathrm{~N}_{3}\right)$, 35.4 (C-6"). $25.3\left(\mathrm{CH}_{3}\right.$. NHAc). $17.0\left(\mathrm{OCH}_{2} \mathrm{CH}_{3}\right)$; MALDITOF MS $651.422 \& 610.466[100,(\mathrm{M}+\mathrm{Na}) \& 40 .(\mathrm{M}+\mathrm{Na})]$.

Acknowledgments. This work was supported by a grant from the Soongsil University Fund, Seoul. Korea. 


\section{References}

1. Salloway. S.: Mermel. L. A.: Seamans. M.: Aspinall. G. O.: Nam Shin, J. E.; Kurjanczyk. L. A.; Penner. I. L. Infect. Immon. 1996, 64. 2945

2. Nam Slin, J. E.: Ackloo. S.: Mainkar. A. S.: Montriro, M. A.: Pang. H.: Penner. J. L: Aspinall. G. O Carbohndr Res. 1998. 305. 223.

3. Aspinall. G. O.: McDonald. A. G.: Raju. T. S.: Pang. H.: Moran. A. P.: Peniner. J. L. Etw J. Biochem. 1993, 213. 1017.

4. Aspinall G. O.: McDonald A. G.: Raju. T. S.: Pang. H.: Kurjanczsk. L. A.: Penner. I. L.: Maran. A. P. Em: J. Biochem. 1993. 231. 1029.

5. Aspinall. G. O.: MeDonald. A. G.: Raju. T. S.: Pang. H.: Mills. S. D.: Kurjanczyk. L. A.: Penner. J. L. J. Bacteriol. 1992. 174. 1324.

6. Aspinall. G. O.; MeDonald A. G.: Pang. H. Carbohndr Res. 1992. 231,13 .

7. Aspinall, G. O: Fujimoto. S: MeDonald. A. G. Pang. $\mathrm{H}$ : Kurjanczyk. L. A.: Penner. J. L. Infect. Inmm. 1994. 62.2122.

8. Yun. M.: Nam Shin. J. E. J. Gradtate School Sontgsil thiv. 1999.

9. Yun. M.: Yoon. S.: Chun. K. H.: Nam Shin. J. E. Agricultural
Biotechology Sint. 1995. 51.

10. Yun1. M.: Yoon1. S.: Shin1. Y.: Chun1. K. H.: Nam Shin. T. E. dirch. Plarm. Res. 2004. 27. 143.

11. Yoon. S.: Shin, Y: Chun. K. H.: Nam Shin. J. E. Bull. Konam Chem. Soc. 2004, 25. 289.

12. Mancuso. A. J.: Swern. D. Symhesis 1981, 165.

13. Veenemant. G. H.: van Boonl. T. H. Tetrohedhon Lett. 1990. 31. 275.

14. Veenemant. G. H.: vant Leeuwent. S. H.: van Boom. I. H. Tetrohedwon Lett. 1990.31. 1331.

15. Yun. M.: Shin. Y; Chun, K. H; Nam Shin. J. E. Bull. Komam Chem. Soc. $2000,21.562$.

16. Yun. M. PhD. Thesis 1999, Part HI. 63.

17. Yun1. M.: Shin. Y.: Yoon1. S.: Chut1. K. H.: Nam Shin1. J. E. Bull. Koredn Chent Soc. 1998. 19. 1239.

18. Leung. O-T.: Douglas. S. P.: Whittield. D. M.: Pang. H. Y. S.: Krepinsky, J. J. Yew. J. Chem 1994, 18, 349.

19. Whittield, D. M.: Pang. H. Carver. J. P.: Krepinsky, J. J. Can. J. Chem. $1990,68,942$.

20. Verduyn. R.: van der Klein. P. A. M.: Douwes. M.: van der Marel. G. A.: van Boom. T. H. Recl. Tron Chim. Pans-Bas 1993. 12 . 464. 